Теорія Ймовір. та Матем. Статист. Вип. 76, 2007
Theor. Probability and Math. Statist.

No. 76, 2008, Pages 33-40 S 0094-9000(08)00729-1

Article electronically published on July 10, 2008

\title{
MARKOV RENEWAL LIMIT THEOREMS
}

UDC 519.21

\author{
S. V. DEGTYAR'
}

\begin{abstract}
We extend the fundamental results of the classical renewal theory to the so-called Markov renewal equation. We prove the Markov renewal theorems for the scheme of series.
\end{abstract}

\section{INTRODUCTION}

The classical renewal theorems describe the asymptotic properties of the convolution

$$
U * g(t)=\int_{0}^{t} U(d s) g(t-s) \quad \text { as } t \rightarrow \infty
$$

where $U$ is the potential of a homogeneous kernel $F(d u)$, which is a probability distribution in a phase space $\left(R_{+}, \mathcal{B}_{+}\right)$.

Without doubt, renewal theory arose from three basic results, namely from theorems due to Feller-Erdös-Pollard, Blackwell, and Smith. Not the last place in renewal theory is taken by the notion of direct integrability introduced by W. Feller. This notion allows one to weaken the assumption on the monotonicity of $g(t)$ in the Smith theorem which resulted in a wide area of applications of renewal theory. We consider the corresponding results for the so-called scheme of Markov renewals.

\section{MAin Results}

Let an abstract measurable space $(E, \mathcal{A})$ be given and let $G(x, d y \times d t)$ be a semihomogeneous kernel in $\left(E \times R_{+}, \mathcal{A} \times \mathcal{B}_{+}\right)$(see [1]), where $\mathcal{B}_{+}$is the Borel $\sigma$-algebra in $R_{+}=[0, \infty)$. Denote by $G(x, d y)$ the base of the kernel $G(x, d y \times d t)$, that is,

$$
G(x, A)=G\left(x, A \times R_{+}\right), \quad x \in E, A \in \mathcal{A} .
$$

Assume that

$$
\sigma \text {-algebra } \mathcal{A} \text { is generated by a finite number of its elements, }
$$

the base $G(x, d y)$ of the kernel $G(x, d y \times d t)$ is conservative [1], and

$$
\text { the Perron root of } G(x, d y) \text { equals } 1 \text {. }
$$

According to a result of [1, $\S 1.2 .1]$, conditions (1) and (2) imply that there exist a nontrivial $\sigma$-finite measure $l$ and a positive $\mathcal{A}$-measurable and $l$-almost everywhere finite

2000 Mathematics Subject Classification. Primary 60K15, 60J25.

Key words and phrases. Markov renewal equation. 
function $h$ such that

$$
\begin{array}{ll}
\int_{E} l(d x) G(x, A)=l(A), & A \in \mathcal{A}, \\
\int_{E} G(x, d y) h(y)=h(x), \quad x \in E \\
\sum_{n \geq 0} G^{n}(x, A)=\infty \quad \text { if } \quad l(A)>0 .
\end{array}
$$

The measure $l$ is unique up to a multiplicative constant, while the function $h$ is unique up to a multiplicative constant and up to the $l$-equivalence.

Consider the Banach space $\mathbb{B}(l, h)$ of complex $\mathcal{A}$-measurable functions $f$ such that

$$
\|f\|=\inf \{c:|f(x)| \leq \operatorname{ch}(x)\}
$$

is finite $l$-almost everywhere. Note that $\|f\|$ is a norm in the space $\mathbb{B}(l, h)$ if one identifies, as usual, the functions coinciding $l$-almost everywhere.

It follows from (3) and (4) that

$$
G f(x)=\int_{E} G(x, d y) f(y), \quad f \in \mathbb{B}(l, h),
$$

defines a linear operator acting from $\mathbb{B}(l, h)$ to $\mathbb{B}(l, h)$.

Consider a family of nonnegative semihomogeneous kernels $G_{\varepsilon}(x, d y \times d t)$ depending on a small parameter $\varepsilon>0$ and assume that $G_{\varepsilon}(x, d y \times d t)$ converges as $\varepsilon \rightarrow 0$ to the kernel $G(x, d y \times d t)$ in the following sense:

$$
\sup _{A \in \mathcal{A}}\left\|\int_{0}^{\infty} G_{\varepsilon}(\cdot, A \times d t) \varphi(t)-\int_{0}^{\infty} G(\cdot, A \times d t) \varphi(t)\right\| \underset{\varepsilon \rightarrow 0}{\longrightarrow} 0
$$

for all continuous bounded functions $\varphi(t), t \geq 0$. In other words, the kernel $G_{\varepsilon}(x, d y \times d t)$ converges to $G(x, d y \times d t)$ "uniformly with respect to $d y$ and weakly with respect to $d t$ in the norm $\|\cdot\|$ evaluated with respect to $x "$.

Denote by $G_{\varepsilon}(x, d y)$ the base of the kernel $G_{\varepsilon}(x, d y \times d t)$. It is shown in 1 that the kernels $G_{\varepsilon}(x, d y \times d t)$ and $G(x, d y \times d t)$ can be represented in the following form:

$$
\begin{aligned}
G_{\varepsilon}(x, d y \times d t) & =G_{\varepsilon}(x, d y) F_{\varepsilon}(x, y ; d t), \\
G(x, d y \times d t) & =G(x, d y) F(x, y ; d t),
\end{aligned}
$$

where the probability distributions $F_{\varepsilon}(x, y ; d t)$ and $F(x, y ; d t)$ are measurable functions of the arguments $x, y \in E$. We assume that

$$
G_{\varepsilon}(x, E) \leq 1 .
$$

Relation (6) implies that

$$
\sup _{A \in \mathcal{A}}\left\|G_{\varepsilon}(x, A)-G(x, A)\right\| \underset{\varepsilon \rightarrow 0}{\longrightarrow} 0 .
$$

Relation (8) is equivalent to the convergence $G_{\varepsilon} \underset{\varepsilon \rightarrow 0}{\longrightarrow} G$ in the operator norm, where the operator $G_{\varepsilon}$ is generated by the kernel $G_{\varepsilon}(x, A)$.

It follows from condition (2) that the norm of the operator $G$ equals one. Moreover, the unity is an isolated simple eigenvalue of the operator $G$ and the unity is an isolated point of the spectrum corresponding to the eigenfunction $h(x)$ in the space $\mathbb{B}(l, h)$.

Assume that

$$
0<\inf _{x \in E} h(x)<\sup _{x \in E} h(x)<\infty
$$


Then the same properties hold for the conjugate operators of $G_{\varepsilon}$ and $G$. The conjugate operators are defined by the following equalities:

$$
\begin{gathered}
\mu G_{\varepsilon}(A)=\int_{E} \mu(d x) G_{\varepsilon}(x, A), \\
\mu G(A)=\int_{E} \mu(d x) G(x, A), \quad A \in \mathcal{A} .
\end{gathered}
$$

Note that the conjugate operators act in the Banach space $\mathbb{M}=\mathbb{M}(E, \mathcal{A})$ of charges $\mu$ of bounded variation. (The full variation is understood as the norm in the space $\mathbb{M}$. The convergence in this norm is equivalent to the uniform convergence for all measurable sets. The unit eigenvalue of the operator $G$ corresponds to the measure $l$ in $\mathbb{M}$.)

The theorem on the semicontinuity of the spectrum (Theorem 3.16 in Chapter 4 of the book [2]) implies that for all sufficiently small $\varepsilon>0$, the operator $G_{\varepsilon}$ has an isolated simple eigenvalue $\lambda_{\varepsilon} \underset{\varepsilon \rightarrow 0}{\longrightarrow} 1$ corresponding to the eigenfunction $h_{\varepsilon}$ in the space $\mathbb{B}(l, h)$ and to the eigenmeasure $\widetilde{l}_{\varepsilon}$ in the space $\mathbb{M}$. Recall that $\lambda_{\varepsilon}$ is the spectral radius. Without loss of generality one can assume that

$$
\begin{gathered}
\int_{E} l(d x) h(x)=1, \quad l(E)=1, \\
\left\|h_{\varepsilon}-h\right\| \underset{\varepsilon \rightarrow 0}{\longrightarrow} 0 .
\end{gathered}
$$

Obviously, the operator $G_{\varepsilon}$ preserves the cone of nonnegative functions $\mathbb{B}_{+}$in the space $\mathbb{B}(l, h)$. Denote by $r_{\varepsilon}$ the spectral radius of $G_{\varepsilon}$ and note that $r_{\varepsilon} \leq 1$ by $(7)$. It is clear that the cone $\mathbb{B}_{+}$is normal and reproducing (see [3]). Thus $r_{\varepsilon}$ is a point of the spectrum of the operator $G_{\varepsilon}$ [3]. This implies that $r_{\varepsilon}=\lambda_{\varepsilon}$ and $h_{\varepsilon}(x) \geq 0$.

Relations (8)-(10) together with the results of [1, 4] imply that there exists a family of measures $l_{\varepsilon}$ in $(E, \mathcal{A})$ that, after an appropriate normalization, are such that

$$
\begin{gathered}
\int_{E} l_{\varepsilon}(d x) G_{\varepsilon}(x, A)=\lambda_{\varepsilon} l_{\varepsilon}(A), \quad A \in \mathcal{A}, \\
\int_{E} l_{\varepsilon}(d x) h_{\varepsilon}(x)=1, \\
\sup _{A \in \mathcal{A}}\left|l_{\varepsilon}(A)-l(A)\right| \underset{\varepsilon \rightarrow 0}{\longrightarrow} 0 .
\end{gathered}
$$

Assume that

$$
0<m=\int_{E} \int_{E} \int_{0}^{\infty} l(d x) G(x, d y \times d t) h(y) t<\infty .
$$

The aim of this paper is to investigate the asymptotic behavior of the convolution

$$
U_{\varepsilon} * g_{\varepsilon}(x, t)=\int_{E} \int_{0}^{t} U_{\varepsilon}(x, d y \times d s) g_{\varepsilon}(y, t-s),
$$

where $U_{\varepsilon}(x, d y \times d t)$ is the potential of the kernel $G_{\varepsilon}(x, d y \times d t)$, while the function $g_{\varepsilon}(x, t)$ is nonnegative and $\mathcal{A} \times \mathcal{B}_{+}$-measurable.

The following result is an analogue of the so-called elementary renewal theorem. Put $\gamma_{\varepsilon}=\left(1-\lambda_{\varepsilon}\right) / m$.

Theorem 1. Let conditions (1)-(2), (6)-(7), (9), and (14) hold. If

$$
\begin{gathered}
\sup _{\varepsilon>0} \sup _{x \in E} \int_{E} \int_{0}^{\infty} G_{\varepsilon}(x, d y \times d t) h(y) t<\infty, \\
\sup _{\varepsilon>0} \int_{E} \int_{E} \int_{T}^{\infty} l(d x) G_{\varepsilon}(x, d y \times d t) h(y) t \underset{T \rightarrow \infty}{\longrightarrow} 0,
\end{gathered}
$$


then

$$
\frac{1}{t} U_{\varepsilon}(x, A \times[0, t]) \underset{\substack{\varepsilon_{\varepsilon \rightarrow c} t \rightarrow 0 \\ \varepsilon \rightarrow 0 \\ t \rightarrow \infty}}{\longrightarrow} \frac{1-e^{-c}}{c} \frac{1}{m} h(x) l(A)
$$

uniformly in $x \in E$ and $A \in \mathcal{A}$.

Proof. For $\operatorname{Im} \alpha \geq 0$, put

$$
\widehat{G}_{\varepsilon}(x, A, \alpha)=\int_{0}^{\infty} e^{i \alpha t} G_{\varepsilon}(x, A \times d t)
$$

and consider the operator

$$
\widehat{G}_{\varepsilon}(\alpha) f(x)=\int_{E} \widehat{G}_{\varepsilon}(x, d y, \alpha) f(y)=\int_{E} \int_{0}^{\infty} G_{\varepsilon}(x, d y \times d t) f(y) e^{i \alpha t} .
$$

It is obvious that the operators $\widehat{G}_{\varepsilon}(i \alpha)$ are real and nonnegative for $\alpha \geq 0$. It is easy to show that

$$
\widehat{U}_{\varepsilon}(i \alpha)=\left[I-\widehat{G}_{\varepsilon}(i \alpha)\right]^{-1}
$$

for $\alpha>0$ and for sufficiently small $\varepsilon>0$. It follows from the limit relation (6) that

$$
\widehat{G}_{\varepsilon}(i \alpha) \underset{\substack{\varepsilon \rightarrow 0 \\ \alpha \rightarrow 0}}{\longrightarrow} G
$$

in the operator norm. Let $\lambda_{\varepsilon}(\alpha)$ be the maximal eigenvalue (spectral radius) of the operator $\widehat{G}_{\varepsilon}(i \alpha)$ that corresponds to the eigenfunction $h_{\varepsilon}(\alpha, x)$ and to the eigenmeasure $l_{\varepsilon}(\alpha, d y)$. Note that

$$
\begin{aligned}
& \lambda_{\varepsilon}(\alpha) \underset{\substack{\varepsilon \rightarrow 0 \\
\alpha \rightarrow 0}}{\longrightarrow} 1, \\
& \left\|h_{\varepsilon}(\alpha)-h\right\| \underset{\substack{\varepsilon \rightarrow 0 \\
\alpha \rightarrow 0}}{\longrightarrow} 0, \quad \sup _{A \in \mathcal{A}}\left|l_{\varepsilon}(\alpha, A)-l(A)\right| \underset{\substack{\varepsilon \rightarrow 0 \\
\alpha \rightarrow 0}}{\longrightarrow} 0 .
\end{aligned}
$$

Without loss of generality one can assume that $h_{\varepsilon}(0, x)=h_{\varepsilon}(x)$ and $l_{\varepsilon}(0, d x)=l_{\varepsilon}(d x)$, where $h_{\varepsilon}$ and $l_{\varepsilon}$ are defined in (10)-(13).

By $C_{1}$ we denote a smooth closed contour in the complex plane that surrounds the point $\lambda_{\varepsilon}(\alpha)$ and whose inner part does not contain any other point of the spectrum of the operator $\widehat{G}_{\varepsilon}(i \alpha)$. By $C_{0}$ we denote a smooth closed contour in the complex plane that surrounds all the points of the spectrum of the operator $\widehat{G}_{\varepsilon}(i \alpha)$ except the point $\lambda_{\varepsilon}(\alpha)$. By the Cauchy formula,

$$
\begin{aligned}
\left(I-\widehat{G}_{\varepsilon}(i \alpha)\right)^{-1} & =\frac{1}{2 \pi i} \oint_{C_{0}}\left(z I-\widehat{G}_{\varepsilon}(i \alpha)\right)^{-1} \frac{d z}{1-z}+\frac{1}{2 \pi i} \oint_{C_{1}}\left(z I-\widehat{G}_{\varepsilon}(i \alpha)\right)^{-1} \frac{d z}{1-z} \\
& =\frac{1}{1-\lambda_{\varepsilon}(\alpha)} \Pi_{\varepsilon}(\alpha)+\frac{1}{2 \pi i} \oint_{C_{0}}\left(z I-\widehat{G}_{\varepsilon}(i \alpha)\right)^{-1} \frac{d z}{1-z}
\end{aligned}
$$

where $i^{2}=-1, \pi=3.14 \ldots$, and the operator $\Pi_{\varepsilon}(\alpha)$ maps the function $f \in \mathbb{B}(l, h)$ to the function $\Pi_{\varepsilon}(\alpha) f(x)=h_{\varepsilon}(\alpha, x) \int_{E} l_{\varepsilon}(\alpha, d y) f(y), x \in E$.

It is also obvious that

$$
\left(z I-\widehat{G}_{\varepsilon}(i \alpha)\right)^{-1} \underset{\substack{\varepsilon \rightarrow 0 \\ \alpha \rightarrow 0}}{\longrightarrow}(z I-G)^{-1}
$$

uniformly in $z \in C_{0}$ in the operator norm. Thus

$$
\frac{1}{2 \pi i} \oint_{C_{0}}\left(z I-\widehat{G}_{\varepsilon}(i \alpha)\right)^{-1} \frac{d z}{1-z} \underset{\substack{\varepsilon \rightarrow 0 \\ \alpha \rightarrow 0}}{\longrightarrow} \frac{1}{2 \pi i} \oint_{C_{0}}(z I-G)^{-1} \frac{d z}{1-z}=I-\Pi,
$$


where the operator $\Pi$ maps the function $f \in \mathbb{B}(l, h)$ to the function

Hence

$$
\Pi f(x)=h(x) \int_{E} l(d y) f(y), \quad x \in E .
$$

$$
\left(I-\widehat{G}_{\varepsilon}(i \alpha)\right)^{-1}-\frac{1}{1-\lambda_{\varepsilon}(\alpha)} \Pi_{\varepsilon}(\alpha) \underset{\substack{\varepsilon \rightarrow 0 \\ \alpha \rightarrow 0}}{\longrightarrow} I-\Pi
$$

in the operator norm. Therefore

$$
\left(1-\lambda_{\varepsilon}(\alpha)\right)\left(I-\widehat{G}_{\varepsilon}(i \alpha)\right)^{-1} \underset{\substack{\varepsilon \rightarrow 0 \\ \alpha \rightarrow 0}}{\longrightarrow} \Pi .
$$

Further we show that

$$
1-\lambda_{\varepsilon}\left(\alpha \gamma_{\varepsilon}\right) \sim \gamma_{\varepsilon}(m+\alpha m)
$$

for a fixed $\alpha>0$. Put $\langle\mu, f\rangle=\int_{E} \mu(d x) f(x)$. By the definitions of $h_{\varepsilon}(\alpha, x)$ and $l_{\varepsilon}(d y)$ we have

$$
\frac{1-\lambda_{\varepsilon}(\alpha)}{\gamma_{\varepsilon}}\left\langle l_{\varepsilon}, h_{\varepsilon}(\alpha)\right\rangle=m\left\langle l_{\varepsilon}, h_{\varepsilon}(\alpha)\right\rangle+\int_{E} l_{\varepsilon}(d x) \int_{E} G_{\varepsilon}(x, d y) \frac{1-\varphi_{\varepsilon}(x, y ; i \alpha)}{\gamma_{\varepsilon}} h_{\varepsilon}(\alpha, y),
$$

where $\varphi_{\varepsilon}(x, y ; \alpha)=\int_{0}^{\infty} e^{i \alpha t} F_{\varepsilon}(x, y ; d t)$.

This implies that the asymptotic equivalence (19) follows from

$$
\lim _{\varepsilon \rightarrow 0} \int_{E} l_{\varepsilon}(d x) \int_{E} G_{\varepsilon}(x, d y) \frac{1-\varphi_{\varepsilon}\left(x, y ; i \alpha \gamma_{\varepsilon}\right)}{\gamma_{\varepsilon}} h_{\varepsilon}\left(\alpha \gamma_{\varepsilon}, y\right)=\alpha m
$$

since

by (15), (17), and

$$
\frac{1-\varphi_{\varepsilon}\left(x, y ; i \alpha \gamma_{\varepsilon}\right)}{\gamma_{\varepsilon}} \leq \alpha \int_{0}^{\infty} t F_{\varepsilon}(x, y ; d t)
$$

$$
\sup _{\varepsilon>0} \sup _{x \in E} \int_{E} G_{\varepsilon}(x, d y) \frac{1-\varphi_{\varepsilon}\left(x, y ; i \alpha \gamma_{\varepsilon}\right)}{\gamma_{\varepsilon}} h_{\varepsilon}\left(\alpha \gamma_{\varepsilon}, y\right)<\infty .
$$

Taking into account the convergence $l_{\varepsilon}(d x) \rightarrow l(d x)$ in variation we see that equality (20) follows from

$$
\lim _{\varepsilon \rightarrow 0} \int_{E} l(d x) \int_{E} G_{\varepsilon}(x, d y) \frac{1-\varphi_{\varepsilon}\left(x, y ; i \alpha \gamma_{\varepsilon}\right)}{\gamma_{\varepsilon}} h_{\varepsilon}\left(\alpha \gamma_{\varepsilon}, y\right)=\alpha m .
$$

Condition (16) means that

$$
\sup _{\varepsilon>0} \int_{E} l(d x) \int_{E} G_{\varepsilon}(x, d y)\left[\mu_{\varepsilon}(x, y)-\frac{1-\varphi_{\varepsilon}(x, y ; i \alpha)}{\alpha}\right] h(y) \underset{\alpha \rightarrow 0}{\longrightarrow} 0,
$$

where $\mu_{\varepsilon}(x, y)=\int_{0}^{\infty} t F_{\varepsilon}(x, y ; d t)$. Moreover the expression in the square brackets on the left hand side of (22) is nonnegative. It is easy to see that (22) and (17) imply

$$
\sup _{\varepsilon>0} \int_{E} l(d x) \int_{E} G_{\varepsilon}(x, d y)\left[\alpha \mu_{\varepsilon}(x, y)-\frac{1-\varphi_{\varepsilon}\left(x, y ; i \alpha \gamma_{\varepsilon}\right)}{\gamma_{\varepsilon}}\right] h_{\varepsilon}\left(\alpha \gamma_{\varepsilon}, y\right) \underset{\alpha \rightarrow 0}{\longrightarrow} 0 .
$$

Thus (21) follows from

$$
\int_{E} l(d x) \int_{E} G_{\varepsilon}(x, d y) \mu_{\varepsilon}(x, y) h_{\varepsilon}\left(\alpha \gamma_{\varepsilon}, y\right) \underset{\varepsilon \rightarrow 0}{\longrightarrow} m .
$$

Considering (17) we see that (23) follows from (16) and (6).

Relations (18) and (19) imply that

$$
\gamma_{\varepsilon} \int_{0}^{\infty} e^{-\alpha \gamma_{\varepsilon} t} U_{\varepsilon}(x, A \times d t) \underset{\varepsilon \rightarrow 0}{\longrightarrow} \frac{1}{1+\alpha} \frac{1}{m} h(x) l(A)
$$

uniformly in $x \in E$ and $A \in \mathcal{A}$. 
Using the continuity theorem for the Laplace transform we get

$$
\gamma_{\varepsilon} U_{\varepsilon}\left(x, A \times\left[0, t / \gamma_{\varepsilon}\right]\right) \underset{\varepsilon \rightarrow 0}{\longrightarrow}\left(1-e^{-t}\right) \frac{1}{m} h(x) l(A)
$$

for all $t \geq 0$ uniformly in $x \in E$ and $A \in \mathcal{A}$, whence Theorem 1 follows.

In what follows we assume that $U_{\varepsilon} * g_{\varepsilon}(x, t)=0$ for $t<0$. For an arbitrary integrable on $(-\infty, \infty)$ function $\omega(s)$, put

$$
\omega * U_{\varepsilon} * g_{\varepsilon}(x, t)=\int_{-\infty}^{\infty} \omega(s) U_{\varepsilon} * g_{\varepsilon}(t-s) d s
$$

Theorem 2. Assume conditions (1)-(2), (6)-(7), (9), (14)-(16), and additionally that the kernel $G(x, d y \times d t)$ is nonlattice. Let $g_{\varepsilon}(x, t) \geq 0$ and

$$
\begin{gathered}
\sup _{\varepsilon>0} \sup _{x \in E} \int_{0}^{\infty} g_{\varepsilon}(x, t) d t<\infty, \\
\sup _{\varepsilon>0} \sup _{x \in E} U_{\varepsilon} * g_{\varepsilon}(x, t)<\infty .
\end{gathered}
$$

If there exists a $\mathcal{B}_{+}$-measurable function $g(t)$ such that

$$
\int_{0}^{\infty}\left|g(t)-\int_{E} l(d x) g_{\varepsilon}(x, t)\right| d t \underset{\varepsilon \rightarrow 0}{\longrightarrow} 0,
$$

then

$$
\lim _{\substack{\gamma_{\varepsilon} t \rightarrow c \\ \varepsilon \rightarrow 0 \\ t \rightarrow \infty}} \omega * U_{\varepsilon} * g_{\varepsilon}(x, t)=e^{-c} \frac{h(x)}{m} \int_{0}^{\infty} g(s) d s \int_{-\infty}^{\infty} \omega(s) d s
$$

uniformly in $x \in E$ for all absolutely integrable on $(-\infty, \infty)$ functions $\omega$.

Proof. By $V_{\varepsilon}(x, d y \times d t)$ we denote the potential of the kernel $\lambda_{\varepsilon}^{-1} G_{\varepsilon}(x, d y \times d t)$. Applying the identity $\lambda_{\varepsilon} U_{\varepsilon}=V_{\varepsilon}+\left(\lambda_{\varepsilon}-1\right) U_{\varepsilon} * V_{\varepsilon}$, we see that the theorem follows from

$$
\omega * V_{\varepsilon} * g_{\varepsilon}(x, t) \underset{\substack{\varepsilon \rightarrow 0 \\ t \rightarrow \infty}}{\longrightarrow} \frac{h(x)}{m} \int_{0}^{\infty} g(s) d s \int_{-\infty}^{\infty} \omega(s) d s .
$$

By condition (25), the convolution $\omega * V_{\varepsilon} * g_{\varepsilon}(x, t)$ can be understood in the sense of the Abel convergence of the corresponding series, that is,

$$
\omega * V_{\varepsilon} * g_{\varepsilon}(x, t)=\lim _{\delta \rightarrow 0} \sum_{k=0}^{\infty}\left(\frac{1-\delta}{\lambda_{\varepsilon}}\right)^{k} \omega * C_{\varepsilon}^{k *} * g_{\varepsilon}(x, t) .
$$

Consider the sequence of operators

$$
\Psi_{\varepsilon}(\alpha)=1-\frac{1}{\lambda_{\varepsilon}} \widehat{G}_{\varepsilon}(\alpha)-\frac{1}{i \alpha \lambda_{\varepsilon}} \Pi_{\varepsilon}(\alpha)\left[G_{\varepsilon}-\widehat{G}_{\varepsilon}(\alpha)\right] .
$$

One can prove that the inverse operator of $\Psi_{\varepsilon}(\alpha)$ exists for all real numbers $\alpha$ if $\varepsilon$ is sufficiently small. Moreover, without loss of generality, one can assume that

$$
\sup _{\varepsilon>0} \sup _{|\alpha| \leq a}\left\|\Psi_{\varepsilon}(\alpha)^{-1}\right\|<\infty
$$

for all $a>0$. Now similarly to the proof of Lemma 3 of Chapter 1 of the book [1] we obtain that

$$
\begin{aligned}
\omega * V_{\varepsilon} & * g_{\varepsilon}(x, t)-\omega * V_{\varepsilon} * g_{\varepsilon}(x,-t) \\
& =\frac{1}{\pi i} \int_{-a}^{a} \sin \alpha t \widehat{\omega}(\alpha)\left[\Psi_{\varepsilon}(\alpha)^{-1} \widehat{g}_{\varepsilon}(x, \alpha)-\frac{1}{i \alpha} \Psi_{\varepsilon}(\alpha)^{-1} \Pi_{\varepsilon} \widehat{g}_{\varepsilon}(x, \alpha)\right] d \alpha
\end{aligned}
$$


for absolutely integrable on $(-\infty, \infty)$ functions $\omega$ such that

$$
\widehat{\omega}(\alpha)=\int_{-\infty}^{\infty} e^{i \alpha t} \omega(s) d s=0 \quad \text { for }|\alpha|>a .
$$

Here

$$
\widehat{g}_{\varepsilon}(x, \alpha)=\int_{0}^{\infty} e^{i \alpha t} g_{\varepsilon}(x, t) d t
$$

and $\Psi_{\varepsilon}(\alpha)^{-1} \widehat{g}_{\varepsilon}(x, \alpha)$ is understood as the action of the operator $\Psi_{\varepsilon}(\alpha)^{-1}$ for the function $\widehat{g}_{\varepsilon}(\cdot, \alpha)$.

Similarly to the proof of Lemma 3 of Chapter 4 in the book [1], one can check that there exists a number $r>0$ such that

$$
\Psi_{\varepsilon}(\alpha)^{-1} \widehat{g}_{\varepsilon}(x, \alpha)=\int_{-\infty}^{\infty} e^{i \alpha t} f_{\varepsilon}(x, t) d t
$$

for $|\alpha| \leq r$, where the functions $f_{\varepsilon}(x, t)$ are measurable with respect to the arguments $x \in E$ and $t \in \mathbf{R}$ and

$$
\sup _{\varepsilon>0} \sup _{x \in E} \int_{-\infty}^{\infty}\left|f_{\varepsilon}(x, t)\right| d t<\infty .
$$

Moreover relation (26) and equality (27) imply that there exists an $\mathcal{A} \times \mathcal{B}$-measurable function $f(x, t)$ such that

$$
\sup _{x \in E} \int_{-\infty}^{+\infty}\left|f_{\varepsilon}(x, t)-f(x, t)\right| d t \underset{\varepsilon \rightarrow 0}{\longrightarrow} 0 .
$$

The rest of the proof is the same as that of the proof of Lemma 3 in [1] mentioned above.

We deduce from Theorem 2 the following analogue of the Blackwell theorem.

Theorem 3. Let the assumptions of Theorem 1 hold. If $G(x, d y \times d t)$ is a nonlattice kernel and there exists $\delta>0$ such that

$$
\inf _{\varepsilon>0} \inf _{x \in E} G_{\varepsilon}(x, E \times[\delta, \infty)) \geq \delta
$$

then

$$
\lim _{\substack{\gamma_{\varepsilon} t \rightarrow c \\ \varepsilon \rightarrow 0 \\ t \rightarrow \infty}} U_{\varepsilon}(x, A \times[t, t+s])=e^{-c} s \frac{h(x)}{m} l(A)
$$

uniformly in $x \in E$ and $A \in \mathcal{A}$ for all $s \geq 0$.

The following result is analogous to the Smith-Feller key renewal theorem.

Theorem 4. Suppose the assumptions of Theorem 2 hold and, in addition, inequality (28) is satisfied,

$$
\int_{E} l_{\varepsilon}(d x) U_{\varepsilon}(x, A \times[t, t+s]) \leq \frac{s+\delta}{\delta^{2}} l_{\varepsilon}(A), \quad A \in \mathcal{A} .
$$

Then if the series $\sum_{k=0}^{\infty} \sup _{k \leq t \leq k+1} g_{\varepsilon}(x, t)$ converges uniformly in $\varepsilon>0$ and $x \in E$ and

$$
\sup _{\varepsilon>0} \delta \int_{E} l(d x) \sum_{k=0}^{\infty}\left\{\sup _{k \delta \leq t \leq k \delta+\delta} g_{\varepsilon}(x, t)-\inf _{k \delta \leq t \leq k \delta+\delta} g_{\varepsilon}(x, t)\right\} \underset{\delta \rightarrow 0}{\longrightarrow} 0
$$

it follows that

$$
\lim _{\substack{\gamma_{\varepsilon} t \rightarrow c \\ \varepsilon \rightarrow 0 \\ t \rightarrow \infty}} U_{\varepsilon} * g_{\varepsilon}(x, t)=e^{-c} \frac{h(x)}{m} \int_{0}^{\infty} g(s) d s
$$

uniformly in $x \in E$. 


\section{CONCLUding REMARKS}

The above renewal theorems have many applications. In particular, applications to the study of final probabilities (in other words, of the limits of transient probabilities) are collected in [1] for the processes with Markov random switchings.

We plan to use renewal theorems in forthcoming papers for studying the asymptotically degenerate sequences of Markov functionals with a countable set of values.

\section{BIBLIOGRAPHY}

1. V. M. Shurenkov, Ergodic Theorems and Related Problems, "Nauka", Moscow, 1989; English transl., VSP International Science Publishers, Utrecht, 1998. MR1690361 (2000i:60002)

2. T. Kato, Perturbation Theory for Linear Operators, Springer-Verlag, Berlin-New York, 1966. MR 0203473 (34:3324)

3. N. Ya. Vilenkin, E. A. Gorin, A. G. Kostyuchenko, S. G. Krasnosel'skiǔ, S. G. Kreйn, V. P. Maslov, B. S. Mityagin, Yu. I. Petunin, Ya. B. Rutitskiı̌, V. I. Sobolev, A. Ya. Stetsenko, L. D. Faddeev, and E. S. Tsitlanadze, Functional Analysis, Second edition, "Nauka", Moscow, 1964; English transl., Wolters-Noordhoff Publishing, Groningen, 1972. MR0390693 $(52: 11516)$

4. N. V. Kartashov, Inequalities in stability and ergodicity theorems for Markov chains with a general phase space. I, Teor. Veroyatnost. i Primenen. 30 (1985), no. 2, 230-240; English transl. in Theory Probab. Appl. 30 (1985), no. 2, 507-515. MR.792617 (87c:60052a)

5. D. Alimov and V. M. Shurenkov, Markov renewal theorems in a scheme of series, Ukrain. Mat. Zh. 42 (1990), no. 11, 1443-1448; English transl. in Ukrainian Math. J. 42 (1990), no. 11, 1283-1288. MR:1098434 (92g:60119)

Department of Higher Mathematics, Vadym Hetman Kyiv National Economic University, Peremogy Avenue, 54/1, Kyiv 03057, Ukraine

Received 6/SEP/2005

Translated by O. I. KLESOV 